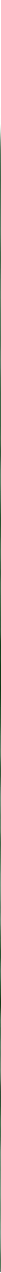

\title{
Lasers and Surface Functionalization
}

\section{How a technology aiming at sustainability is spreading and supporting laser development in Europe}

\section{Francesca Moglia, Jose Pozo and Andrés F. Lasagni}

Nature shows how special nano- and micrometer-sized features can render surfaces hydrophobic or anti-bacterial. Laser-based texturing is a technique capable of imitating this and functionalizing surfaces without the use of additives. EU-funded projects, such as LAMpAS, aim at achieving high throughputs with systems based on kW-level, short and ultrashort-pulsed lasers, enabling this technology for the competitive manufacturing and commercialization of domestic appliances with new features and fast-moving consumer products.

Laser-based surface treatment as defined spans a wide range of specific applications and the appropriate laser technologies. For example, if required just for marking, only relatively low power is needed (typically below $20 \mathrm{~W}$ ). Nevertheless, it can approach the kilowatt power level, depending on the material and type of treatment to be carried out. The same applies to the nature and the operation of the lasers: depending on the specific application, they can be $\mathrm{CO}_{2}$, solid-state, excimer or diode lasers and can operate in cw or pulsed mode with different pulse durations and repetition rates.

This flexibility of the market as well as the reliability and development potential of laser technology have motivated various groups to exploit it further to support sustainable solutions for the global market.
Currently, industry demands highly value-added products offering new features at a low-cost. Some of these can allow for direction-dependent fluid transport, antibacterial/antifouling effects, anti-reflectance, friction reduction, self-cleaning and water repellency. Often, to obtain these features, only the surface of the object is affected, being treated with external composites or spread with coatings. In addition, this 
Fig. 2 Image of a lotus leaf, which is naturally superhydrophobic due to the combination of micro- and nano-structures on its surface (left) and SEM picture of the same surface (right, source: W. Barthlott, lotus-salvinia.de)

process usually needs to be periodically renewed and implies the use of chemicals and additives.

\section{The capability of nature}

Observing nature, it is possible to find many different examples in which characteristics of certain surfaces result from intrinsic features in the nano/micrometer scale. For example, the hydrophobic properties, which minimize adhesion of water droplets to some leaves and the wings of certain insects, are caused by surface micro- and nano-patterns resembling those shown in Fig. 1 and 2. The surface of a lotus leaf is full of micro-meter sized pillars, which contain hundreds of nanometer-sized ripples and the combination of these structures prevents water from reaching the surface. The geometry of the surface is the only origin of these characteristics. The same is valid for the butterfly shown in Fig. 3. In this case, no pigmentation is present on its wings surface, just its special topography allows for reflectance of only blue light.

\section{Commercializing natural prop- erties}

Imitating these and other similar natural examples, surfaces of industrial products can be directly functionalized obtaining the same characteristics in a more environmentally friendly way then adding external composites. With this respect, laser surface texturing has shown to be capable of obtaining such advanced functionalities and the best results come from laser sources operating at short and ultrashort pulse dura-
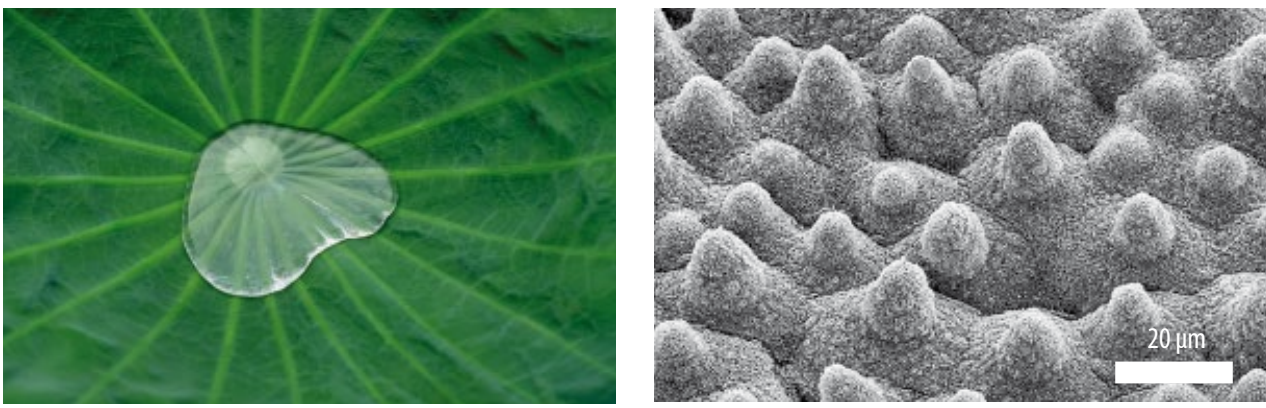

tions, i.e. at nano- down to pico- and femtosecond. Furthermore, for commercialization of a complete laser-surface texturing system, the whole process needs to undergo an enhancement of efficiency, flexibility and productivity resulting in high throughput.

\section{Recognition by the European Commission and the potential of the LAMpAS project}

In recent years, the potential of laser technology has been recognized also by the European Commission within the Horizon 2020 Framework Program and, jointly through a Public Private Partnership (PPP) with the European Technology Platform Photonics21, substantial investments have been made. Together with the environmentally sustainable aim of structure functionalization, laser technology is at the heart of many current EU-funded projects.

One of these projects is called LAMpAS, which is currently running under Grant Agreement 825132 and the co-ordination of the Technische Universität Dresden. With eight partners, among them Next Scan Technology, New Infrared Technologies and Lasea, this project has the challenging aim of building a complete laser-based surface-texturing system reaching both high resolution (feature sizes $200 \mathrm{~nm}-200 \mu \mathrm{m}$ ) and high throughput (up to $1-5 \mathrm{~m}^{2}$ / $\mathrm{min}$ ), to address both the functionality and the commercialization purposes, respectively. This is possible thanks to a multi-beam processing technique with interference patterns on large spots, called direct laser interference patterning (DLIP), delivered to the material by high-speed polygon scanners. These technologies, if thoroughly applied in controlling the interaction between laser light and the substrate, can also stimulate self-organization processes generating laser-induced periodic surface structures (LIPSS) in a homogeneous way. These result in a three-level hierarchical surface pattern: a micrometer-scaled structure, derived directly by DLIP, plus two distinct, overlapped, sub-micrometer sized LIPSS patterns called high and low spatial frequency LIPSS (HSFLs and LSFLs, respectively), making artificial surfaces resembling natural ones and mirroring their fascinating properties, as mentioned at the beginning of this text. Two examples of such artificially generated patterns are shown in Fig. 4.

All this is applicable to different kinds of surfaces (polymers, metals, ceramics, ...) and obtainable in LAMpAS thanks to the efforts of Trumpf Laser, a project partner that is concentrating on the development of a think-disk based, ps-laser, delivering up to $1.5 \mathrm{~kW}$ at $\mathrm{MHz}$-level repetition rate. These parameters are the result of a tested ideal scenario whereby a high pulse energy in the millijoule range at a moderate repetition rate of $10 \mathrm{MHz}$ or less, combined with a scan speed of $100 \mathrm{~m} / \mathrm{s}$ or more, enables large spot sizes with an interference pattern, giving a high feature size resolution.

Two end-users, and partners of the projects, are Bosch for pharmaceutical
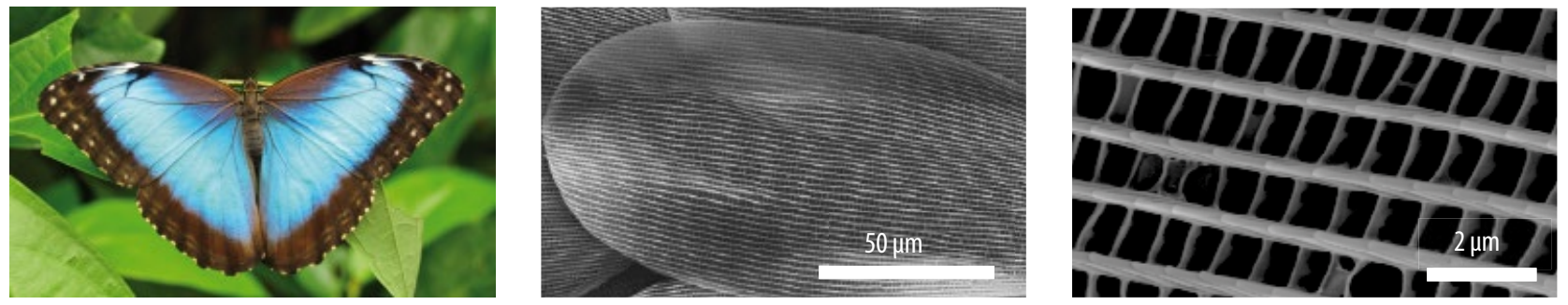

Fig. 3 Image of the Morpho Peleides butterfly, whose color is due to a periodic variation of the surface topography on the micrometer scale that reflects only blue light (Source: Zoe Schnepp; CC BY-SA 4.0). 

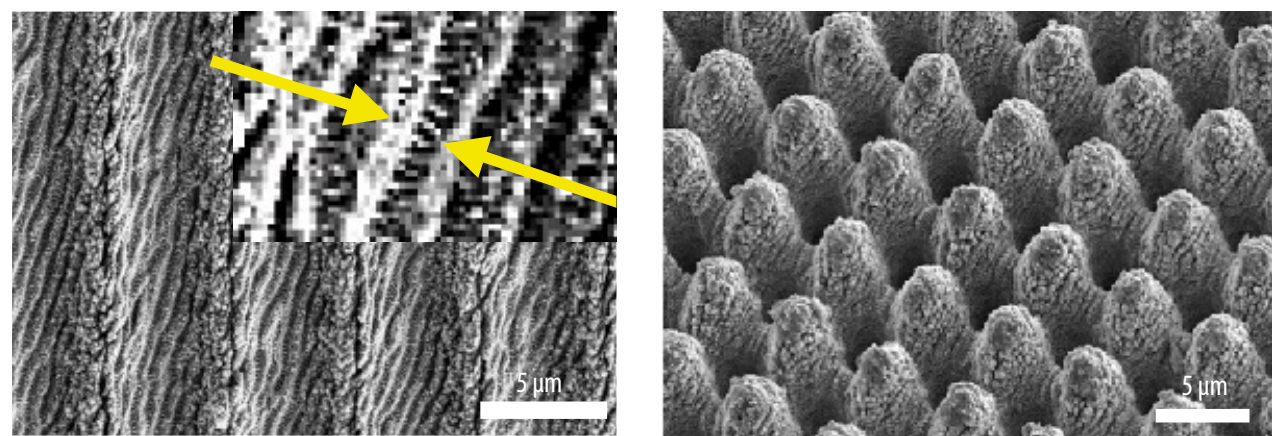

Fig. 4 Three-level hierarchical surface pattern induced by direct laser interference patterning (DLIP) with $\mu \mathrm{m}$-scaled features (left main picture) overlapped with two different orders of laser-induced periodic surface structures (LIPSS) in the order of $800 \mathrm{~nm}$ and less than $150 \mathrm{~nm}$ (visible in the left inset). The pillar-like structure (right) was also generated with DLIP. A ps-laser was used for both. (Source: S. Aguilar-Morales et al., Mater. Process. Technol. 252 (2018) 313-321; CC BY-NC-ND 4.0).

packaging and $\mathrm{BSH}$ for domestic appliance products. One of their aims is to test a robust industry-oriented prototype, able to functionalize surfaces in real time and increase the value of their products by adding anti-fingerprint, easy-to-clean and antibacterial features and new decoration finishes.

One of the overall aims of the project is to have a total improvement in product performance greater than $20 \%$, based on the deployed surface functionalities.

\section{Other EU-funded projects in laser-based surface functional- ization}

The potential of a mature technology running at longer pulse duration is the one exploited by other EU-funded projects such as PROMETHEUS. It is coordinated by EWF (European Federation for Welding, Joining and Cutting) and counts on partners such as Prima Power, Photonics Bretagne, HOLO/OR, AIMEN Centro Tecnologico and the Fraunhofer IWS. They aim at applying DLIP with a pulsed-laser architecture working in the nanosecond-regime, with average power $>1 \mathrm{~kW}$ and repetition rates $>500 \mathrm{~Hz}$ to serve end users in different areas, such as automotive and fast-moving consumer goods markets.

Other projects besides LAMpAS aim at exploiting the technology of ultra-fast lasers in the ps-range. Two of them are called TresClean and Laser4Surf. The first is coordinated by the Universitá
This content reflects only the the European Commission and Photonics 21 are not responsible for any use that may be made of the information it contains. authors' view and has already tested experimentally and numerically in previous projects (e.g. LASER4FUN). Laser4Surf is co-ordinated by Ceit-IK4is supported, among others, by Multitel, and also bases its future platform for laser surface texturing on similar laser systems. Nevertheless, they focusing on other end-user fields such as the increase of product performance of advanced batteries, linear encoders and medical components.

When the pulse duration is shortened further to the femtosecond level, the multi-beam processing techniques described above can still be used if the parameters of the lasers are adjusted accordingly. The EU-funded FEMTOSURF project is coordinated by Femtika and is investigating this alternative with the support of Amphos, who is developing a laser with a duration of a few hundreds of femtoseconds, based on their InnoSlab amplification technology, reaching a $2-3 \mathrm{~kW}$ laser power level, with a similar aim of reaching a throughput higher than $1 \mathrm{~m}^{2} / \mathrm{min}$. Among others, end-users in the fields of healthcare, ship building and aerospace wish to profit from a final product providing laser-structured surfaces with anti-bacterial, self-cleaning, frictionreduced and anti-icing properties.

LAMpAS has received funding from the European Union's Horizon 2020 research and innovation programme under grant agreement No [825132) and is an initiative of the Photonics Public Private Partnership.

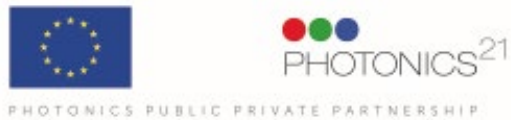

\section{Authors}

Francesca Moglia is a project leader at EPIC, the European Photonics Industry Consortium. She has a background in crystal growth, spectroscopy, optics and laser development for research and large research facilities. Before joining EPIC, she moved from R\&D to scientific management for the coordination of a consortium of funding agencies in the field of astroparticle physics. She graduated in Physics from the University of Pisa in Italy, acquired her PhD (Dr. rer. nat.) at the Universität Hamburg in the field of Laser Physics and pursued her post-doctoral activities at DESY, Deutsches Elektronen-Synchrotron in Hamburg, Germany.

\begin{abstract}
Jose Pozo is director of technology and innovation at EPIC, and as such he represents more than four hundred leading corporate members active in the field of photonics. His work consists of actively engaging with them and providing them with the tools to strengthen their position in the supply chain. He has twenty years' background in photonics technology and market knowledge, and a large network within the industrial and academic photonics landscape. Jose holds a PhD in electrical engineering from the University of Bristol, UK, and a MSc and BEng in telecom engineering from Spain and Belgium.
\end{abstract} degli Studi di Parma, which is supported by partners such as as Alphanov and University of Stuttgart, and pursues a similar strategy to LAMpAS in employing thin-disk based ps-lasers for DLIP and LIPPS. These are more oriented in testing different laser wavelengths and parameters, which the LAMpAS community
Further author: Prof. Dr. Andrés F. Lasagni, Technische Universität Dresden and Fraunhofer-Institut für Werkstoff- und Strahltechnik - IWS

Dr. Francesca Moglia, Dr. Jose Pozo, EPIC - European Photonics Industry Consortium, Rue Hamelin 17, Paris, France; e-mail: francesca.moglia@epic-assoc.com, jose.pozo@epic-assoc.com, Web: www.epic-assoc.com 